\title{
Pautas orientativas para la asistencia a la diversidad y la inclusión de personas sordas
}

\section{Guidelines for diversity assistance and inclusion of deaf people}

DOI: $10.46932 / \mathrm{sfjdv2n4-013}$

Received in: Jun1st, 2021

Accepted in: Sep 30th, 2021

\author{
Roberto García Sánchez \\ Doctor en Historia de la Psiquiatría \\ Profesor de Psicología de la Personalidad \\ Universidad de La Laguna \\ Campus de Guajara, s/n, 38071 San Cristóbal de La Laguna, Santa Cruz de Tenerife \\ E-mail: extrgarcias@ull.edu.es \\ Justo Pedro Hernández González \\ Doctor en Medicina y Cirugía \\ Profesor de Historia de la Medicina \\ Universidad de La Laguna \\ Aparcamiento, Campus de Ofra, s/n, 38071 San Cristóbal de La Laguna, Santa Cruz de Tenerife \\ E-mail: jhdezj@ull.edu.es
}

\section{RESUMEN}

Comunidad Sorda es aquella que participa de unos valores culturales y lingüísticos construidos en torno a la lengua de signos y a una concepción visual del mundo.

Entre las personas sordas usuarias de la lengua de signos algunas aprendieron a signar en su infancia y otras siendo ya adultas; hay quienes son usuarias de audífonos o implantes cocleares y, entre ellas, hay quienes usan la lengua de signos y quienes no.

También debemos mencionar a aquellas personas sordas que, a causa de un sistema educativo no inclusivo, tienen problemas de expresión y comprensión de textos escritos.

Al igual que en el resto de la población, entre las personas sordas encontraremos niños, jóvenes, mayores, personas sordas con otra(s) discapacidad(es)... Todas y cada una de ellas con sus necesidades y demandas concretas. Es importante saber que, aun tratándose de un colectivo heterogéneo, todas las personas sordas, cualquiera que sea su tipo o grado de sordera, situación individual e independientemente de que sean o no usuarias de las lenguas de signos, comparten la necesidad de acceder a la comunicación e información del entorno sin barreras de ningún tipo.

Por ese motivo es necesario desarrollar un servicio de orientación, asesoramiento y acción tutorial específico para el alumnado sordo que tenga en cuenta sus necesidades y dificultades y que evite cualquier tipo de discriminación o falta de accesibilidad al contenido universitario del tipo que sea. Por lo tanto, es necesario proporcionar este servicio con los recursos audiovisuales necesarios, intérpretes de lengua de signos española y formación continua a la comunidad universitaria. Es fundamental coordinarse con las asociaciones de personas sordas para cumplir los requisitos básicos que garanticen su inclusión, puesto que éstas son las que conocen mejor sus necesidades por la lucha de sus derechos, y orientar a la universidad para la consecución de dicha finalidad.

Palabras clave: personas sordas, lengua de signos, inclusión. 


\begin{abstract}
A Deaf Community is one that participates in cultural and linguistic values built around sign language and a visual conception of the world.

Among the deaf people who used sign language, some learned to sign in their childhood and others when they were adults; there are those who use hearing aids or cochlear implants and, among them, there are those who use sign language and those who do not.

We will also find deaf people who, because of a non-inclusive educational system, have problems of expression and comprehension of written texts.

As in the rest of the population, among the deaf people we will find children, young people, elderly, deaf people with other disability(ies). . . Each and every one of them with their specific needs and demands. It is important to know that, even if it is a heterogeneous collective, all deaf people, whatever their type or degree of deafness, individual situation and regardless of whether or not they are users of sign languages, share the need to access the communication and information of the environment without barriers of any kind.

For this reason it is necessary to develop a service of guidance, advice and specific tutorial action for deaf students that takes into account their needs and difficulties and avoids any type of discrimination or lack of accessibility to university content of any kind. Therefore, it is necessary to provide this service with the necessary audiovisual resources, Spanish sign language interpreters and continuing education to the university community. It is essential to coordinate with associations of deaf people to meet the basic requirements to ensure their inclusion, since they are the ones who best know their needs by fighting for their rights, and guide the university to achieve that goal.
\end{abstract}

Keywords: deaf people, sign language, inclusion.

\title{
1 INTRODUCCIÓN
}

El movimiento inclusivo surge con fuerza defendiendo que los centros educativos deben satisfacer las necesidades de todos los alumnos, sean cuales sean sus características personales, psicológicas o sociales. La inclusión se vincula al acceso, la participación y los logros de todos los alumnos (Opertti, 2008). Implica transformar la cultura, la organización y las prácticas de los centros para atender a la diversidad de necesidades educativas de todos los alumnos. Es una enseñanza adaptada al estudiante, cuyas acciones van dirigidas a eliminar o minimizar las barreras físicas, personales o institucionales que limitan las oportunidades de aprendizaje, el acceso y la participación en las actividades formativas. Por eso, la opción educativa basada en un modelo inclusivo implica un desarrollo global que exige cambios que involucran a la totalidad del sistema educativo, en dimensiones como las de crear culturas, políticas y prácticas inclusivas (Villar y Alegre, 2008). Por tanto se considera que uno de los indicadores de calidad de la universidad radica en cómo responde a la diversidad de los alumnos, a la vez que refleja la excelencia docente de los profesionales vinculados al mismo (Alegre, 2009).

García y Cotrina (2011) proponen el desarrollo de un diseño universal de aprendizaje como práctica pedagógica en el contexto de la educación superior, de manera que se pueda mejorar la respuesta socio-educativa y los andamiajes de la accesibilidad, las tecnologías de la información y la comunicación, 
la inclusión y sostenibilidad curricular en procesos de innovación docente que sustenten el nuevo modelo social de universidad. Como señala Sosa (2009), la inclusión en la universidad se puede abordar desde el punto de vista ético (la inclusión es un derecho humano fundamental), sociológico (la discapacidad como construcción social y la inclusión como modo de dar voz a las personas con discapacidad), organizativo (plantea los cambios que hay que introducir en la organización universitaria para avanzar hacia una institución más inclusiva) y pedagógico-docente (es preciso promover en la universidad sistemas pedagógico-docentes que faciliten la permanencia y la promoción de los alumnos con discapacidad).

En las aulas universitarias cada vez es más numeroso el colectivo de personas con algún tipo de discapacidad que accede a las mismas, pero este hecho queda oscurecido dado que la universidad no ha seguido un proceso claro de adaptación a medidas especiales de inclusión.

Una de las claves para que la inclusión en la universidad sea posible, es que el profesorado universitario desarrolle la capacidad para atender a la diversidad y alcanzar la competencia intercultural e inclusiva (Alegre y Villar, 2006). Desde el ángulo abierto de la interculturalidad y la inclusividad, los educadores deben considerar que la diversidad y la igualdad han de dejar de ser inapresables y uno de los cambios más importantes que se están necesitando es el de ayudar a crear, elaborar y promover un discurso de la diversidad (Shi-Xu, 2001; Voss y Bufkin, 2011).

Leyser, Zeiger y Romi (2011) aplicaron una escala de autoeficacia docente que permitió cotejar la intensidad formativa sobre deficiencias de escolares en tres grupos de alumnos universitarios en formación según su pertenencia a un grupo general o especial. El entrenamiento en el área de estudiantes con necesidades educativas especiales, comparado con estudiantes con poco o ningún entrenamiento, dio como resultado una eficacia en la promoción de puntuaciones significativamente superiores en los cuatro factores de autoeficacia (que eran las variables dependientes: eficacia docente, eficacia personal, eficacia social y eficacia de bajo rendimiento).

En los sistemas de garantía de calidad de las universidades recogidas actualmente en los protocolos de evaluación de las titulaciones, se han de contemplar diversas medidas para favorecer la incorporación a la vida universitaria de los estudiantes con discapacidad, desarrollando un sistema de orientación y tutoría adaptado a sus necesidades educativas específicas, que dé respuesta a los problemas para su integración a la vida universitaria (Abad, Álvarez y Castro, 2008).

La implantación de un sistema de orientación y tutorías en el marco de la enseñanza universitaria para todo el alumnado en general, y para los que poseen algún tipo de discapacidad en particular, constituye un factor de la calidad orientado a la mejor integración de todo el alumnado, la optimización del proceso formativo, la promoción de una mayor igualdad de oportunidades, la potenciación del principio de orientación a lo largo de la vida, el refuerzo de la educación inclusiva, la prevención del 
abandono de los estudios, entre otros beneficios (Rodríguez, 2004; Álvarez, Asensio, Forner y Sobrado, 2006).

Como resaltan Alonso y Díez (2007), la creación del Espacio Europeo de Educación Superior exige a las Universidades el cumplimiento de una serie de estándares de calidad y la atención a los estudiantes con discapacidad es uno de ellos. Esto se debe traducir en medidas de atención a los universitarios con discapacidad para asegurar y garantizar un acceso igualitario, una actuación equitativa y prácticas adecuadas que den consistencia a una verdadera educación superior de calidad. En este sentido, en el Real Decreto 1393/2007 que ordena las enseñanzas universitarias oficiales, recoge referencias claras a la accesibilidad y a la educación para todos, como principios básicos a tener en cuenta a la hora de diseñar los nuevos títulos universitarios, de modo que el acceso y el tránsito de los estudiantes con discapacidad por este nivel formativo se lleve a cabo de forma adecuada y satisfactoria.

Para poder lograr la inclusión debemos eliminar las etiquetas tradicionales relacionadas con la discapacidad y reforzar los apoyos específicos para una universidad inclusiva.

Las dificultades del alumnado con necesidades específicas derivadas de su discapacidad a la hora de enfrentarse a la transición hacia la enseñanza universitaria se agudizan, puesto que los problemas que tienen para integrarse y desarrollar su proyecto formativo son, por lo general, mayores que los del resto de sus compañeros. Esto se debe a que, probablemente, sea el paso más conflictivo por todos los cambios que implica y la transición más compleja que debe afrontar por las diferencias tan marcadas respecto a las etapas previas (Fernández y García, 2002; Eckes y Ochoa, 2005).

Muchas Universidades aún no están preparadas para "recibir" a estos alumnos, no sólo en lo que a estructuras físicas o arquitectónicas se refiere, sino también en lo que respecta a su regulación normativa y a los aspectos estrictamente curriculares o de organización docente (Susinos y Rojas, 2003). Como señala Tolrà (2002), es evidente que se están haciendo esfuerzos considerables para dar continuidad a las medidas de apoyo y asesoramiento que requiere el alumnado con discapacidad, pero todavía se está lejos de la consolidación de los principios de igualdad de oportunidades y no-discriminación de estas personas en el contexto universitario.

Para resolver estas situaciones se hace necesario avanzar en el diseño, aprobación y difusión de normativas que aseguren la adecuada atención y apoyo al alumnado con discapacidad, en las que se concreten unos estándares de calidad e indicadores de buenas prácticas en el funcionamiento de los servicios de apoyo a la discapacidad en los diferentes contextos, que permitan establecer puentes de conexión entre etapas para darle continuidad a la formación (Alcantud, Ávila y Asensi, 2000; Forteza y Ortego, 2003; CERMI, 2005; Luque y Rodríguez, 2008). 
Los procesos de Convergencia Europea permiten flexibilizar la docencia universitaria y racionalizar los planes de estudios, a la vez que nos hace a todos más sensibles a los cambios que beneficiarán a las personas con diferencias. Por ejemplo, se tiende a una organización de la actividad docente centrada en las actividades de los estudiantes, una mayor incidencia de la acción tutorial y la convivencia entre formas presenciales y no presenciales (Álvarez, 2002; Alegre, 2004; Castro y Alegre, 2009). Los programas de orientación y tutoría universitaria constituyen un elemento fundamental para favorecer la calidad y la inclusión de los estudiantes con discapacidad, ya que permiten desarrollar el carácter integrador en la educación superior (Bayot, Rincón y Hernández, 2002; Luque y Rodríguez, 2005). Para todos los estudiantes, pero especialmente para aquellos que tienen alguna discapacidad, la orientación puede ser una vía importante para introducir medidas compensatorias que den respuesta a sus necesidades específicas y poner en práctica una atención individualizada que permita que estos estudiantes se integren y promocionen (Álvarez, 2012).

\section{OBJETIVOS}

El objetivo principal de este trabajo consiste en la propuesta de creación de un servicio de orientación, asesoramiento y acción tutorial para el alumnado sordo que se enmarque dentro del servicio actual con el que cuenta la Universidad de La Laguna, partiendo de las necesidades específicas y dificultades con las que cuenta esta población universitaria y garantizando de este modo su adecuado proceso de aprendizaje.

\section{METODOLOGÍA}

La creación de un servicio de orientación, asesoramiento y acción tutorial específico para el alumnado sordo tiene como objetivo garantizar el apoyo durante el acceso a la Universidad y permanencia en el periodo de estudios. Esto se consigue a partir de una atención integral y personalizada, evaluándose las necesidades académicas especiales del alumno con discapacidad auditiva. Es necesario que éste conozca el servicio y lo tenga como referente para disponer de los recursos y programas que se desarrollan.

Entre los recursos disponibles, el estudiante con discapacidad auditiva debe contar con lo siguiente: asesoramiento y orientación, prioridad en la elección de grupo y turno, tutorías especializadas, adaptaciones de materiales docentes, adaptación en las pruebas de evaluación, diseño de la modalidad de la prueba, recursos técnicos necesarios para su realización, aumento de tiempo para la realización de exámenes, Intérprete de Lengua de Signos Española (ILSE), reserva de los primeros asientos en las filas delanteras del aula e información escrita (guión o texto) en caso de usar material audiovisual, crear la 
figura del Estudiante Colaborador (entre sus tareas podemos mencionar el apoyo en la toma de apuntes, en el acceso a la información, ...), servicios especiales en bibliotecas (información bibliográfica personalizada, reserva de sitio, ampliación de préstamo de libros...), entre otros. Será estrictamente necesaria la formación directa al profesorado y al personal de administración y servicios. Respecto al ILSE es necesario que haya un supervisor experto en lengua de signos (una persona sorda especialista en lengua de signos o a través de alguna entidad representante por personas sordas) que pueda valorar la adecuación de los intérpretes al puesto de trabajo. Este es un criterio importante que hay que tener en cuenta ya que algunos entran a asumir la profesión tras varios años sin ejercer como tal.

Como recursos técnicos se empleará el uso de grabadoras digitales, libretas autocopiativas, sistemas de FM o bucles magnéticos, material audiovisual con subtitulado y signado, residencias universitarias con habitaciones adaptadas (sistema luminoso de emergencia y aviso). Apoyo técnico personalizado con la colaboración del Servicio de Informática para proporcionar los recursos específicos que necesita el estudiante con discapacidad auditiva.

La página web, además, deberá ser accesible al disponer cada servicio un enlace de la información en lengua de signos realizada por un Especialista de Lengua de Signos Española (ELSE). También es necesario generar un blog público donde se puedan compartir experiencias y opiniones de mejora para dichos alumnos, generando una comunicación directa e inmediata con el servicio de orientación de la universidad garantizando la transparencia, puesto que es un blog de acceso público.

Si el alumno lo desea, puede optar por el programa de tutorización realizado por compañeros de la misma carrera de cursos superiores, y por el programa de acompañamiento en el que puede contar con la ayuda de un compañero para tomar apuntes o para realizar cualquier gestión vinculada con la Universidad, en esto consistiría la figura antes mencionada del Estudiante Colaborador. Es importante también el desarrollo de la figura del profesor tutor cuyas funciones son las de solventar los problemas que le surgen al alumno, de asesorarle y de servir de conexión entre los demás profesores.

También se dispondrá de una "Guía de orientación a profesores de estudiantes con discapacidad auditiva", en la que se facilitan una serie de consejos prácticos para una mejor comunicación e integración, centrado en tres pilares:

- Alumnos preuniversitarios: donde incluya un protocolo de actuación para la atención de las necesidades especiales en las pruebas de acceso.

- Coordinadores de los centros universitarios: presenta el protocolo de actuación del servicio con los centros universitarios. 
- Alumnos de nuevo ingreso: incorporando una guía de acogida donde se informa de las ayudas, becas y cuestiones relativas a la atención de las necesidades especiales.

Para un correcto uso de esta guía será necesaria la colaboración de una entidad representativa de personas sordas donde se realice una charla y formación adecuada en materia de sensibilización sobre la comunidad sorda destinada a profesores, pero también a compañeros y personas que tengan contacto habitual con los alumnos sordos.

\section{RESULTADOS}

Nos encontramos ante una propuesta epistémica que pretende alcanzar unos resultados ante una situación de futuro, por lo tanto dichas conclusiones se consideran en un ámbito estrictamente prospectivo.

Sin embargo, esperamos encontrar resultados similares a los arrojados por el Informe Sumario de evidencias sobre educación inclusiva de 2017, que citaremos a continuación, y que ha sido publicado por el Instituto Alana y elaborado por la Escuela de educación de posgrado de Harvard y la firma mundial Abt Asociados.

Éste realiza una revisión de toda la investigación existente sobre educación inclusiva, concretamente comprende la revisión de 280 estudios realizados en 25 países. El informe define entornos educativos inclusivos de acuerdo a la Observación General n ${ }^{\circ} 4$ del Comité sobre los derechos de las personas con discapacidad, es decir, aquellos que garantizan «la participación plena y efectiva, la accesibilidad, la asistencia y el buen rendimiento académico de todos los alumnos». Se reconoce que la proliferación de prácticas educativas inclusivas deriva del creciente reconocimiento de que los estudiantes con discapacidad prosperan cuando se les garantizan las mismas oportunidades educativas y sociales que a los estudiantes sin discapacidad. También pone de relieve las importantes barreras que suponen las actitudes culturales negativas y las ideas erróneas existentes sobre la educación inclusiva entre la comunidad educativa.

Este informe concluye, entre sus hallazgos más importantes, que existen "evidencias claras y consistentes de que los entornos educativos inclusivos generan importantes beneficios, a corto y largo plazo, para los estudiantes con y sin discapacidad". Cuando son adultos, los alumnos con discapacidad tienen mayor probabilidad de cursar estudios en post secundaria, de conseguir un empleo y vivir de forma independiente. 
Además, los estudiantes con discapacidad educados en aulas ordinarias superan a sus pares educados en aulas segregadas. Cuanto mayor es la proporción de la jornada escolar que los alumnos con discapacidad pasan con estudiantes sin discapacidad, mejores son los resultados en matemáticas y lengua.

La participación en entornos inclusivos, además, produce beneficios sociales y emocionales para los estudiantes con discapacidad, incluyendo la formación y el mantenimiento de relaciones positivas entre compañeros, que tienen implicaciones importantes para el aprendizaje y el desarrollo psicológico.

Con respecto a los estudiantes sin discapacidad, se describen cinco beneficios de la inclusión para los mismo: reducción del miedo a la diferencia humana, acompañado de un mayor grado de comodidad y conciencia (menos miedo a las personas que miran o se comportan de manera diferente); crecimiento en la cognición social (mayor tolerancia hacia los demás, comunicación más efectiva con todos los compañeros); mejoras en el concepto de sí mismo (aumento de la autoestima, percepción de la condición social y el sentido de pertenencia); desarrollo de principios morales y éticos personales (menos prejuicios, mayor receptividad a las necesidades de los demás); y amistades cálidas y afectuosas.

\section{CONCLUSIONES}

Es una propuesta de futuro, no es actual, por lo que no hay datos de referencia, lo cual supone una dificultad añadida. Damos por hecho que es un tema no solucionado y por eso se propone su puesta en práctica.

Se espera que después de este trabajo haya un clima que favorezca ciertos resultados de respuesta en esta línea epistémica, pues se considera algo imprescindible para garantizar la correcta inclusión y consecución de los derechos de las personas sordas.

Podemos mencionar los resultados del trabajo de Álvarez, Alegre y López (2012), que va en la línea de la presente propuesta:

Encontraron que en el período preuniversitario el alumnado encuestado manifestó haber recurrido poco al orientador escolar (60,2\%), al profesor de apoyo a las necesidades educativas especiales (84,5\%), al intérprete de lengua de signos $(96,1 \%)$ o al asistente personal $(90,3 \%)$. Llama la atención que aproximadamente la mitad de los encuestados (50,5\%) señalara que no habían recibido información y orientación relativa a los estudios universitarios. Ante las necesidades específicas que tienen estos estudiantes, la respuesta educativa de muchos profesores no se ajusta a los principios de la educación inclusiva, lo que dificulta su proceso formativo y el logro de metas personales, académicas y profesionales.

De la totalidad de estudiantes consultados, el 53,4\% consideró que la Universidad de La Laguna no disponía de los recursos adecuados para hacer frente a las necesidades del alumnado con discapacidad. 
Concretamente, los estudiantes señalaron que la Universidad debería mejorar los apoyos al alumnado con discapacidad en cuanto a "la mejora de acceso a las aulas" (P6), la "orientación profesional" (P13), la “orientación psicológica y pedagógica" (P22) y el "carácter más empático del profesorado" (P85). En este sentido, los estudiantes demandaban un programa de atención a estudiantes con discapacidad en el que se desarrollaran aspectos relacionados con la orientación escolar $(r=.454 ; \mathrm{p}<0.000)$, con el conocimiento de la vida universitaria $(\mathrm{r}=.436 ; \mathrm{p}<0.000)$ y con la definición de su itinerario académicoprofesional ( $\mathrm{r}=.489 ; \mathrm{p}<0.000)$.

La existencia de un programa de tutorías específico para los estudiantes con discapacidad fue valorada positivamente por 74,8\% del alumnado encuestado, apuntando la necesidad de que sería necesario crear un programa de estas características en su Facultad o Centro, desde el que se pudiese orientar y apoyar a este colectivo de estudiantes.

En otro estudio realizado por Mesa (2013) se llega a las siguientes conclusiones:

Es necesario realizar un estudio sobre la percepción que tiene la familia del alumno con discapacidad auditiva, sobre la evolución de su familiar-alumno en el sistema educativo y los apoyos recibidos; también debe hacerse un estudio transversal con la finalidad de analizar la evolución de la respuesta educativa que recibe; sería apropiado estudiar las medidas que se están llevando a cabo con los alumnos con discapacidad auditiva; es muy importante realizar estudios sobre el grado de desarrollo del trabajo colaborativo, entre los diferentes profesionales docentes y no docentes y otros servicios comunitarios implicados; analizar los planes de formación continua que se están llevando a cabo, evaluar la calidad de esta formación y el nivel de implicación de los propios profesores en el compromiso de su formación continua. Por último, se debería elaborar un estudio sobre las condiciones acústicas de las aulas universitarias.

Teniendo en cuenta lo observado múltiples fuentes de información y, basándonos en nuestro trabajo, que aquí se ha presentado, podemos extraer las siguientes conclusiones:

Se plantea necesaria la creación de un Servicio de Intérpretes de Lengua de Signos Española, contratado por la Universidad, como personal de servicios universitario, formando parte de la comunidad universitaria y no como servicio externalizado, que formaría parte del servicio de orientación, asesoramiento y acción tutorial.

Sería altamente recomendable que todos los profesores universitarios, así como personal de limpieza, comedor... recibieran una formación completa para aprender a relacionarse y comunicarse de forma correcta con personas sordas o alguna discapacidad auditiva.

Convendría que el servicio de orientación y atención que se propone para su creación, tuviera las competencias para visionar el contenido que se imparte en las facultades de la Universidad de La Laguna 
y determinar la accesibilidad o no de éste, para su posterior corrección y asesoramiento a profesores, en caso de ser necesario.

Otra conclusión importante que extraemos es que, al ser un servicio especializado, debería tener todas las competencias pertinentes para resolver problemas de accesibilidad u orientación por parte de estos alumnos. Ello implica no solamente contar con un intérprete sino realizar las acciones necesarias para dotar de recursos técnicos a los alumnos sordos, tanto en las aulas de su correspondiente facultad como en la residencia universitaria, si fuera necesario.

Debemos resaltar que al ser un servicio universitario debería, por un lado, realizar encuestas de satisfacción al finalizar cada curso para poder ampliar mejoras para cursos sucesivos y, por otro lado, participar en las labores de investigación propias de las universidades para dar visibilidad, conocimiento y valor a este colectivo.

Es sumamente importante que un servicio especializado cuente con el asesoramiento de los órganos o instituciones de relevancia de este colectivo para garantizar así un servicio completo y no meras conjeturas de lo que debería ser lo correcto. También serviría para acercar la universidad al propio colectivo de personas sordas.

La conclusión más importante que podemos inferir es que hay elementos que faltan o no se están cumpliendo respecto a las medidas que debería tomar la universidad en esta materia, con profesores, alumnos, recursos técnicos... y que se deberían incorporar. Si a ello le sumamos los efectos altamente positivos de una educación inclusiva, según todos los datos de investigación, entenderemos cómo nuestra propuesta sólo debería poder obtener resultados igualmente positivos. Por lo tanto, con la creación de este servicio se podría dar una respuesta uniforme, contundente y satisfactoria a todas las cuestiones relacionadas con accesibilidad, orientación, recursos técnicos de todo tipo, resolución de conflictos, mediación, herramientas comunicativas... Todo ello implica concentrar esta labor en una unidad con competencia suficiente, evitando así que las personas afectadas busquen constantemente formas de solucionar sus conflictos enviando correos multitudinarios porque no saben a quién dirigirse, o bien, se han dirigido ya a muchas personas y no han obtenido respuesta o resolución del hecho.

Por último, podemos afirmar que con esta propuesta se solventaría todos los problemas de accesibilidad a los que se enfrentan las personas sordas, garantizando así la plena inclusión. 


\section{REFERENCIAS BIBLIOGRÁFICAS}

Abad, M., Álvarez, P. y Castro, J. (2008). Apoyo a la integración de estudiantes con discapacidad en la enseñanza universitaria: algunas medidas y propuestas de actuación orientadora. Educación y Diversidad: Anuario Internacional de Investigación sobre Discapacidad e Interculturalidad, 2, 129-150.

Alana y ABT Associates (2016). A summary of the evidence on inclusive education. Estados Unidos: Hehir, T., Grindal, T., Freeman, B., Lamoreau, R., Borquaye, Y. y Burke, S.

Alcantud, F.; Ávila, V. y Asensi, M. (2000). La integración de estudiantes con discapacidad en los estudios superiores. Servicio de publicaciones: Universidad de Valencia.

Alegre, O.M. (2004). Atienda a la diversidad del alumnado universitario. En L. M. Villar Angulo (Coord.), Programa para la mejora de la docencia universitaria. Madrid: Pearson-McGraw Hill.

Alegre, O.M. y Villar, L.M. (2006). Online Faculty Development in the Canary Islands: A Study of EMentoring. Higher Education in Europe, 3 (1), 65-81.

Alegre, O.M. y Villar, L.M. (2009). Evaluation of a 10-year special education masters's degree program: the case of La Laguna University. International Journal of Special Education, 24 (2), 116-129.

Alegre, O.M. y Villar, L.M. (2009). Master's Degree “Education in Diversity” (MDED): Toward Inclusion Education Quality. International Journal of Training and Learning in Higher Education, 22 (1), $1-13$.

Alonso, A. y Diéz, E. (2008). Universidad y Discapacidad: indicadores de buenas prácticas. Siglo Cero. Revista Española sobre Discapacidad Intelectual, vol. 39 (2), n 226, pp. 82-98.

Álvarez, P. (2002). La función tutorial en la Universidad: una apuesta por la mejora de la calidad de la enseñanza. Madrid: EOS.

Alvarez, P. Alegre, O.M. y López, D. (2012). Las dificultades de adaptación a la enseñanza universitaria de los estudiantes con discapacidad: un análisis desde un enfoque de orientación inclusiva. RELIEVE, v. 18, n. 2, art. 3. DOI:10.7203/relieve.18.2.1986.

Bayot, A.; Rincón, B. \& Hernández, F. (2002). Orientación y atención a la diversidad: descripción de programas y acciones en algunos grupos emergentes. RELIEVE, 8 (1), 66-87. Recuperado de http://www.uv.es/RELIEVE/v8n1/RELIEVEv8n1_2.htm

Castro, J. y Alegre, O (2009). Alumnado con discapacidad en la Universidad. Guías para el profesorado. Tenerife: Turquesa.

CERMI (2006): Guía de recursos para alumnos con discapacidad. Investigación Educativa. Sevilla: Colás, $\mathrm{M}^{\mathrm{a}}$. y Buendía, L.

Eckes, S. y Ochoa, T. (2005). Students with disabilities: transition from high school to higher education. American Secondary Education, 33 (3), 1-16. 
Fernández, J. y García, C. (2002): Transición de estudiantes con necesidades educativas de la educación secundaria a la Universidad. De la integración a una educación para todos: la atención a la diversidad desde la Educación primaria a la Universidad. Madrid: CEPE.

Forteza, D. y Ortego, J. (2003). Los servicios y programas de apoyo universitarios para personas con discapacidad. Estándares de calidad, acción y evaluación. Revista de Educación Especial, 33, 9-26.

García, M. y Cotrina, M. (2011). Accesibilidad, inclusión e innovación docente en la Universidad. Quaderns digitals: Revista de Nuevas Tecnologías y Sociedad, 67, 1-12.

Leyser, Y., Zeiger, T., y Romi, S. (2011). Changes in Self-efficacy of Prospective Special and General Education Teachers: Implication for inclusive education. International Journal of Disability, Development and Education, 58 (3), 241-255.

Luque, D. y Rodríguez, G. (2005). Accesibilidad y adaptaciones curriculares al alumnado con discapacidad en la Universidad. Una reflexión docente. I Congreso Nacional de Universidad y Discapacidad, Salamanca.

Luque, D. y Rodríguez, G. (2008). Alumnado universitario con discapacidad: elementos para la reflexión psicopedagógica. REOP, 19 (3), 270-281.

Mesa, J.L. (2013). La percepción sobre la inclusión del alumnado con discapacidad auditiva en la Educación Secundaria, Educación Superior y enseñanzas de régimen especial en Gran Canaria (tesis doctoral). Universidad de Las Palmas de Gran Canaria, Gran Canaria, España.

Opertti, R. y Brady, J. (2011). Developing inclusive teachers from an inclusive curricular perspective. Prospects, 41 (3), 459-472.

Rodríguez, S. (2004). Manual de tutoria universitaria. Barcelona: Octaedro.

Shi-Xu (2001). Critical Pedagogy and Intercultural Communication: Creating discourses of diversity, equality, common goals and rational-moral motivation. Journal of Intercultural Studies, 22 (3), 279-293.

Susinos, T. y Rojas, S. (2003). Los servicios de apoyo universitarios y la orientación para el acceso a la Universidad. Revista de Educación Especial, 33, 27-38.

Tolrá, M. J. (1998). Retos de los servicios universitarios de apoyo a la integración. XVII Seminario Interuniversitario de Teoría de la Educación "La Educación Universitaria: nuevos retos educativos y tecnológicos". Málaga.

Voss, J.A., y Bufkin, L.J. (2011). Teaching All Children: Preparing Early Childhood Preservice Teachers in Inclusive Settings. Journal of Early Childhood Teacher Education, 32 (4), 338-354.

Villar, L. M. y Alegre, O. M. (2008). Measuring Faculty Learning in Curriculum and Teaching competence (CTC). On line Courses. Interactive learning Environments, 16. 